\title{
Studies of cosmic ray anisotropies with DAMPE
}

\section{Shijun Lei, ${ }^{a, *}$ Enrico Catanzani, ${ }^{b}$ Wei Jiang, ${ }^{a}$ Chuan Yue ${ }^{a}$ and ${ }^{*}$ on behalf of the DAMPE Collaboration}

(a complete list of authors can be found at the end of the proceedings)

${ }^{a}$ Key Laboratory of Dark Matter and Space Astronomy, Purple Mountain Observatory, Chinese Academy of Sciences, Nanjing 210023, China

${ }^{b}$ Istituto Nazionale di Fisica Nucleare (INFN) - Sezione di Perugia, I-06123 Perugia, Italy

E-mail: sjlei@pmo.ac.cn, enrico.catanzani@pg.infn.it, jiangwei@pmo.ac.cn, yuechuan@pmo.ac.cn

\begin{abstract}
A small anisotropy in the arrival directions of comic rays has been consistently observed by ground detectors based on very large sample of events. The Dark Matter Particle explorer (DAMPE) has so far accumulated more than 10 billion events above $\mathrm{GeV}$ with relatively high spatial and energy resolution during its 5-year plus on-obit operation, providing a good data sample for probing the anisotropy of cosmic rays. We introduce in this proceeding our optimizations in the direction measurement, data sampling and anisotropy analysis. The anisotropy predicted by the east-west effect due to the Earth magnetic field and the Compton-Getting effect due to the Earth revolution are then applied to the validation of our analysis.
\end{abstract}

$37^{\text {th }}$ International Cosmic Ray Conference (ICRC 2021)

July 12th-23rd, 2021

Online - Berlin, Germany

\footnotetext{
*Presenter
} 


\section{Introduction}

Although the arrival direction of charged cosmic rays (CRs) at near the Earth is highly isotropized by the interstellar/intergalactic magnetic field, a small $\left(O 10^{-4}-10^{-3}\right)$ anisotropy in the CR flux has been unambiguously detected by various ground-based instruments (e.g., see [16]), shedding light on the origin and propagation of the CRs [7, 8], which but remains an open question of fundamental significance in astrophysics. Comparing to the ground-based instruments, the space-born detectors bear some unique advantages in probing the CR anisotropy. For example, the space detectors usually cove a wider sky area and are sensitive to CR anisotropy in both R.A. and decl, while the ground instruments are only sensitive to CR anisotropy in R.A. Also, many space detectors have the ability of particle discrimination and hence being able to measure the anisotropy of different CR components. Indeed, a number of attempts in probing the CR anisotropy have been carried out by several space detectors, like PAMILA [13], AMS-02 [12], and Fermi [9-11]. However, no CR anisotropy has been explicitly detected by the space detectors, believed mainly due to the much limited acceptance of the space detectors. Nevertheless, certain revealing constrains on the CR anisotropy have been provided by the studies, together with an effective methodology in doing the CR anisotropy analysis, which lays a solid foundation for future works of this kind. We introduce in this work our studies of the CR anisotropy based on the 5-year observation of the Dark Matter Particle Explorer.

\section{The DAMPE Detector}

The Dark Matter Particle Explorer (DAMPE) is a space-born high-energy cosmic-ray and gamma-ray detector successfully launched on December 17, $2015[15,16]$. The DAMPE satellite has been operating smoothly ever since in a survey mode, i.e., with the detector top always pointing to the local zenith, in a sun-synchronous orbit at the altitude of $500 \mathrm{~km}$ with an inclination of $97.4^{\circ}$. Scanning the entire sky twice every year with a wide instantaneous field of view ( $>1.0 \mathrm{sr}$ ), DAMPE accumulates more than 10 billion $\mathrm{CR}$ events above $\mathrm{GeV}$ up to now, forming an excellent data sample for probing the CR anisotropy.

The DAMPE payload consists of four sub-detectors. The plastic scintillation detector (PSD) measures the absolute charge of incident CR particles and acts as anti-coincidence shield for gamma-rays. The silicon-tungsten Tracker (STK) measures the direction of incident particles with relatively high precision. The $\mathrm{BGO}$ calorimeter (BGO) also measure the $\mathrm{CR}$ particle direction but with relatively low precision, along with the capability of energy measurement and electron/hadron discrimination. The neutron detector provides an independent measurement that helps enhance the electron/hadron identification.

\section{Event Selection}

Direction measurement of the incident CR particles is the foundation of CR anisotropy analysis. As shown in Fig. 1 for example, with proper track reconstruction, both the STK and the BGO could measure the direction of incident CR particles. The STK with much finer structure usually provides a better measurement of the direction with a resolution of $\sim 0.1^{\circ}[18]$. The point spread function of 


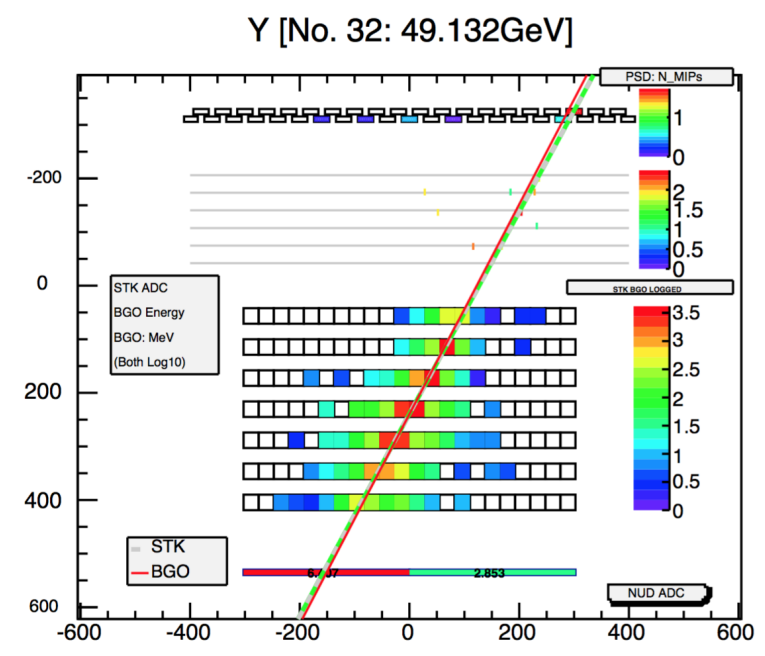

Figure 1: Tracks reconstructed for a typical simulated proton event. While the STK track reconstructed (grey line) usually measures the real direction of the primary particle (green dash line) with a typical resolution of $\sim 0.2^{\circ}$, there is the possibility that a track produced by secondary/back splash particles being wrongly taken. The BGO track (red solid line) reconstructed using the entire BGO shower profile is but more reliable within the uncertainty of $\sim 5.0^{\circ}$.

the STK direction measurements, however, has a non-negligible tail extended to fairly large angle due to the fact that a track caused a secondary/back splash particle in the STK could be wrongly taken as the primary one. On the other hand, the BGO measurement, although with a moderate resolution of $\sim 5.0^{\circ}$ [19], is reconstructed using the entire shower profile in the BGO and hence more reliable within the uncertainty. In this analysis, we thus combine the STK and the BGO measurement to form an optimized estimation of the direction by taking the best STK direction measurement that is within $15^{\circ}$ of the $\mathrm{BGO}$ measurement.

For the time period of the data, we adopt the DAMPE data of five years, from April 2016 to April 2021. The data of an integer number of years are used to mitigate the possible influence of the Compton-Getting effect [20], which largely cancels out for a whole year in our anisotropy analysis in the Equatorial/Galactic coordinate. The Compton-Getting effect is most significant in a specially chosen coordinate so that the direction of the Earth revolution is fixed. We check in Sec. 3 the Compton-Getting effect in this special coordinate as a benchmark to validate our method of anisotropy analysis.

The geomagnetic field could be a big trouble in the anisotropy analysis as it causes an undesired anisotropy especially in relative low energy band. The energy and the incident angle of the CR particles must be carefully chosen to mitigate the effect of the geomagnetic field. And here we use the famous "east-west" effect to determine the cuts in the energy and incident angle of the CR particles, as positively charged particles arriving from near the horizon from the east are blocked by the Earth because their trajectories bend downward into the atmosphere. The "east-west" effect is expected to be most significant at the earth shadowing angle $\theta=112^{\circ}$ given the $500 \mathrm{~km}$ orbit high of the satellite. But our track reconstruction can not discriminate the two possible directions 

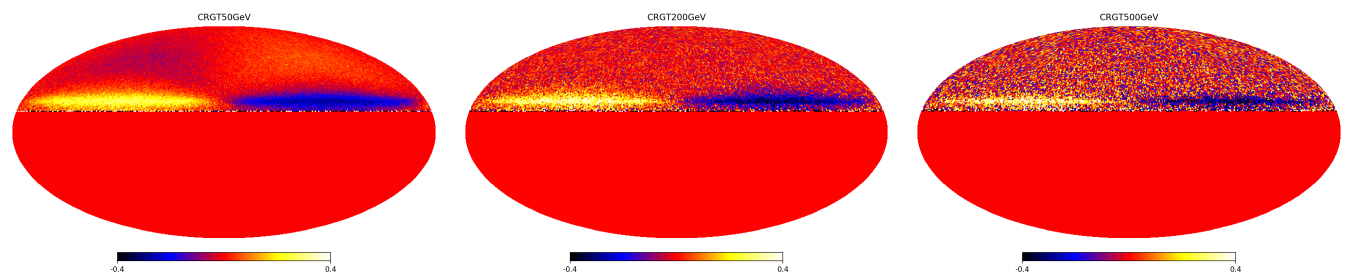

Figure 2: Sky maps in altitude-azimuth coordinates for CR samples of different low energy cuts. The "east-west" effect is clearly visible in for the CR sample with BGO energy $>50 \mathrm{GeV}$ at all altitude angle (which is equivalent to the incidental angle $\theta$ in detector coordinate in our case given the survey mode of DAMPE). The "east-west" effect fades out at small altitude with increasing energy, and concentrate on the area centered at $\theta=68$ due to the Earth shadowing and inverted particle direction. See test for more details.

of a straight line, and wrongly takes all the bottom-up events as top-down ones, we may expect a pattern caused by the "east-west" effect to show up at $\theta=68^{\circ}$ and in the opposite way. Indeed, as shown in Fig. 2, in the altitude-azimuth coordinate, structures caused by the "east-west" effect is clearly seen even for the CR sample with BGO energy $>500 \mathrm{GeV}$ (the energy deposit in the BGO calorimeter) if no incident angle cut is applied. But the anisotropy structures concentrates on large incident angle (given the survey mode of the DAMPE satellite prior mentioned, the altitude angle in the map is the incident angle $\theta$ in the detector coordinate) with increasing CR energy. To ensure a minimum quality at this step, we imply an energy cut of BGO energy (hereafter simply energy) $>100 \mathrm{GeV}$, which typically accounts about $40 \%$ of the intrinsic rigidity of the incident particle, and an incidental angle cut of $\theta<45^{\circ}$. The so chosen energy and incident angle cuts for the CR particles also guarantee that no bottom-up events are included in our further analysis, as they are safely blocked by the Earth.

With all the above mentioned event selection criteria, we obtain a CR sample of 44.4 million with energy $>100 \mathrm{GeV}$. We also do the anisotropy analysis for CR samples of higher energies, and with sample volumes decreasing in a typical power law.

\section{Reference Map}

A key issue in the anisotropy analysis is to produce the reference map that is expected to represent the detector's observation of an ideally isotropic sky. A comparison between the real map and the reference map may then reveal the anisotropy we desired, by eliminating the variation in the exposure that is much more significant than the possible anisotropy. Following [11], we used the "event-rate" method to generate the reference map. This method makes use of a time-averaged event rate and the response of the detector, together with the live-time and the pointing history of the satellite for this purpose. Before we go through the standard procedure in doing the anisotropy analysis, however, we want to first check the stability of the detector response, which is a basic assumption of the method for producing an effective reference map but not necessarily true.

We demonstrate in Fig. 3 the time variation of the detector response by comparing those in different time periods. As clearly seen, the variation of the detector response is limited to the scale of $\sim 10^{-3}$ for two consecutive one-year periods, while the difference increase to about $\sim 4 \times 10^{-3}$ for two periods 4 years apart. To mitigate the long time variation of the detector response, we also 

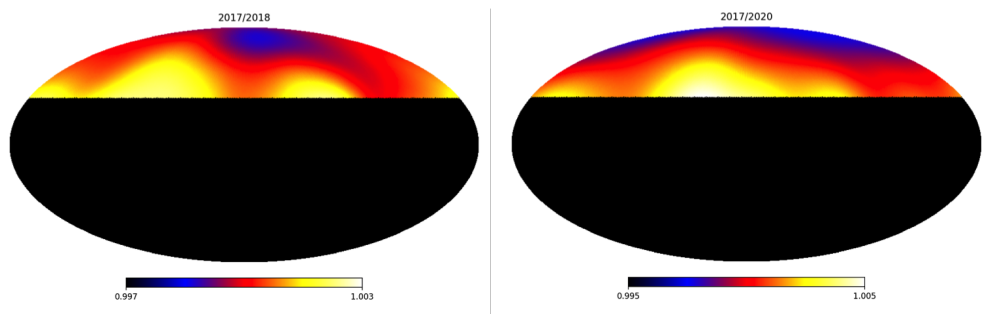

Figure 3: Comparison of the detector response. The left (right) map is produce by dividing the response map of the year 2017 to that of the year 2018 (2020). Difference of scale $\sim 10^{-3}$ is seen in the left map, and that of $\sim 4 \times 10^{-3}$ in the right one. The general consistency of the pattern in the two maps indicates that the difference is real but not simply random fluctuation.

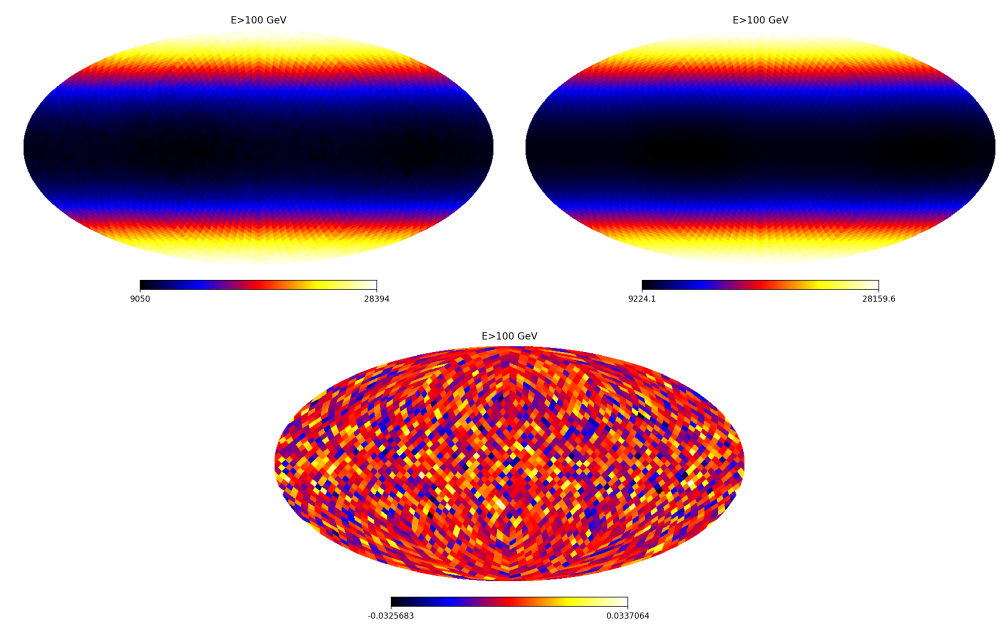

Figure 4: Data (top-left) and reference sky map (top-right) in equatorial coordinate (J2000) for all events in the date set. A comparison of the two maps results into the sky map of relative intensity (bottom). All the maps are created using HELPix pixelization scheme with 3072 pixels.

apply a year-long bin in generating the reference maps following [11]. And we believe that the time dependent variation in the detector response within one year is smaller than $<10^{-3}$ and may introduce a systematic anisotropy to the final result of $<10^{-3}$.

Following standard procedure, we obtain the data map and the reference map, as shown in Fig. 4 for the CR sample with BGO energy $>100 \mathrm{GeV}$. A comparison of the two map resulted into the map of relative intensity also shown in the figure.

The relative intensity map was then decomposed using the spherical harmonic analyzing tool anafast in the HELPix library [21]. We find that the angular power spectrum calculated for the relative intensity map is consistent with the null hypothesis, i.e., isotropic sky. Following [11], we also calculated a $95 \%$ CL upper limit of $\delta_{U L}=1.2 \times 10^{-3}$.

\section{Results and Conclusion}

We form a carefully chosen CR data sample with BGO energy $>100 \mathrm{GeV}$ based on the 5-year plus observation of DAMPE. Our analysis is consistent with the null hypothesis, i.e., isotropic sky 
and provide a $95 \% \mathrm{CL}$ upper limit of $\delta_{U L}=1.2 \times 10^{-3}$ on the dipole amplitude at a minimum BGO energy of $100 \mathrm{GeV}$.

\section{Acknowledgement}

We appreciate the very helpful comments from a number of colleagues, especially Yi Zhang and Qiang Yuan in the methodology and validation of the anisotropy analysis.

The DAMPE mission is funded by the strategic priority science and technology projects in space science of Chinese Academy of Sciences. This work is supported by the Astronomy Joint Grant U1631111 and the Science Grant 12073087.

\section{References}

[1] Abdo, A. A., et al., ApJ, 698, 2121, 2009

[2] Amenomori, M., et al., ApJ, 836, 153, 2017

[3] Aab, A., et al., Sci, 357, 1266, 2017

[4] Bartoli, B., et al., ApJ, 861, 93, 2018

[5] Abeysekara, A. U., et al.,ApJ, 871, 96, 2019

[6] Soldin, D. et al, PoS (ICRC2019), 14, 2019

[7] Liu, W. et al, JCAP, 10, 10, 2019

[8] Qiao, B. Q. et al, JCAP, 12, 7, 2019

[9] Ackermann, M., et al., PRD, 82, 2010

[10] Abdollahi, S., et al., PRL, 118, 1103, 2017

[11] Ajello, M., et al., ApJ, 883, 33, 2019

[12] Gebauer, I., et al. PoS (ICRC2017), 186, 2017

[13] Adriani, O. et al., arXiv, 180110310, 2018

[14] Munoze, M., et al., PoS(ICRC2019), 113, 2019

[15] J. Chang, et al., CJSS, 34, 550, 2014

[16] J. Chang et al., Aph, 95, 6, 2017

[17] Ambrosi, G., et al., Aph, 106,18, 2019

[18] Tykhonov, A., et al., NIMPR, 924, 309, 2019

[19] Lei, S. J., et al., PoS (ICRC2019), 329, 2019

[20] Compton, A. H., and Getting, I. A., Phys. Rev., 47 817, 1935

[21] Gorski, K. M., et al., ApJ, 622,759, 2005 


\section{Full Authors List: DAMPE Collaboration}

F. Alemanno ${ }^{1,2}$, Q. An ${ }^{3,4}$, P. Azzarello ${ }^{5}$, F. C. T. Barbato ${ }^{1,2}$, P. Bernardini ${ }^{6,7}$, X. J. Bi ${ }^{8,9}$, M. S. Cai ${ }^{10,11}$, E. Casilli6, ${ }^{6}$, E. Catanzani ${ }^{12}$, J. Chang ${ }^{10,11}$, D. Y. Chen ${ }^{9,10}$, J. L. Chen ${ }^{13}$, Z. F. Chen ${ }^{10,11}$, M. Y. Cui ${ }^{10}$, T. S. Cui ${ }^{14}$, Y. X. Cui ${ }^{10,11}$, H. T. Dai ${ }^{3,4}$, A. De Benedittis ${ }^{6,7}$, I. De Mitri ${ }^{1,2}$, F. de Palma ${ }^{6,7}$, M. Deliyergiyev ${ }^{5}$, M. Di Santo ${ }^{6,7}$, Q. Ding ${ }^{10,11}$, T. K. Dong ${ }^{10}$, Z. X. Dong ${ }^{14}$, G. Donvito ${ }^{15}$, D. Droz ${ }^{5}$, J.

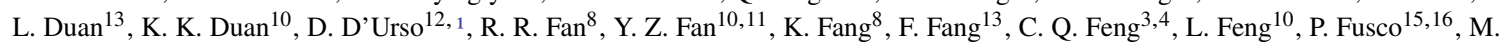

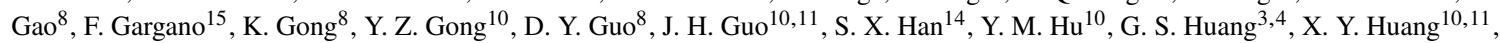
Y. Y. Huang ${ }^{10}$, M. Ionica ${ }^{12}$, W. Jiang ${ }^{10,11}$, J. Kong ${ }^{13}$, A. Kotenko ${ }^{5}$, D. Kyratzis ${ }^{1,2}$, S. J. Lei ${ }^{10}$, W. H. Li ${ }^{10,11}$, W. L. Li ${ }^{14}$, X. Li ${ }^{10}$, X. Q.

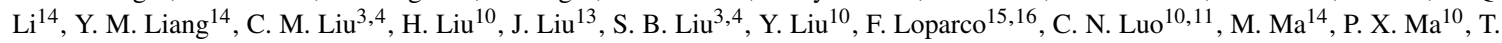
$\mathrm{Ma}^{10}$, X. Y. Ma ${ }^{14}$, G. Marsella ${ }^{6,7,2}$, M. N. Mazziotta ${ }^{15}$, D. Mo ${ }^{13}$, X. Y. Niu ${ }^{13}$, X. Pan ${ }^{10,11}$, A. Parenti ${ }^{1,2}$, W. X. Peng ${ }^{8}$, X. Y. Peng ${ }^{10}$, C. Perrina ${ }^{5,3}$, R. Qiao ${ }^{8}$, J. N. Rao ${ }^{14}$, A. Ruina ${ }^{5}$, M. M. Salinas ${ }^{5}$, G. Z. Shang ${ }^{14}$, W. H. Shen ${ }^{14}$, Z. Q. Shen ${ }^{10}$, Z. T. Shen ${ }^{3,4}$, L. Silveri ${ }^{1,2}$, J. X. Song ${ }^{14}$, M. Stolpovskiy ${ }^{5}$, H. Su ${ }^{13}$, M. Su${ }^{17}$, H. R. Sun ${ }^{3,4}$, Z. Y. Sun ${ }^{13}$, A. Surdo ${ }^{7}$, X. J. Teng ${ }^{14}$, A. Tykhonov ${ }^{5}$, H. Wang ${ }^{14}$, J. Z. Wang ${ }^{8}$, L. G. Wang ${ }^{14}$, S. Wang ${ }^{10,11}$, S. X. Wang ${ }^{10,11}$, X. L. Wang ${ }^{3,4}$, Y. Wang ${ }^{3,4}$, Y. F. Wang ${ }^{3,4}$, Y. Z. Wang ${ }^{10}$, D. M. Wei ${ }^{10,11}$, J. J. Wei ${ }^{10}$, Y. F. $\mathrm{Wei}^{3,4}$, D. $\mathrm{Wu}^{8}$, J. Wu ${ }^{10,11}$, L. B. Wu ${ }^{1,2}$, S. S. Wu ${ }^{14}$, X. Wu ${ }^{5}$, Z. Q. Xia ${ }^{10}$, E. H. Xu ${ }^{3,4}$, H. T. Xu ${ }^{14}$, Z. H. Xu ${ }^{10,11}$, Z. L. Xu ${ }^{10}$, Z. Z. Xu ${ }^{3,4}$, G. F. Xue ${ }^{14}$, H. B. Yang ${ }^{13}$, P. Yang ${ }^{13}$, Y. Q. Yang ${ }^{13}$, H. J. Yao ${ }^{13}$, Y. H. Yu ${ }^{13}$, G. W. Yuan ${ }^{10,11}$, Q. Yuan ${ }^{10,11}$, C. Yue ${ }^{10}$, J. J. Zang ${ }^{10,4}$, F. Zhang ${ }^{8}$, S. X. Zhang ${ }^{13}$, W. Z. Zhang ${ }^{14}$, Y. Zhang ${ }^{10}$, Y. J. Zhang ${ }^{13}$, Y. L. Zhang ${ }^{3,4}$, Y. P. Zhang ${ }^{13}$, Y. Q. Zhang $^{10}$, Z. Zhang ${ }^{10}$, Z. Y. Zhang ${ }^{3,4}$, C. Zhao ${ }^{3,4}$, H. Y. Zhao ${ }^{13}$, X. F. Zhao ${ }^{14}$, C. Y. Zhou ${ }^{14}$, and Y. Zhu ${ }^{14}$

${ }^{1}$ Gran Sasso Science Institute (GSSI), Via Iacobucci 2, I-67100 L'Aquila, Italy

${ }^{2}$ Istituto Nazionale di Fisica Nucleare (INFN) -Laboratori Nazionali del Gran Sasso, I-67100 Assergi, L'Aquila, Italy

${ }^{3}$ State Key Laboratory of Particle Detection and Electronics, University of Science and Technology of China, Hefei 230026, China

${ }^{4}$ Department of Modern Physics, University of Science and Technology of China, Hefei 230026, China

${ }^{5}$ Department of Nuclear and Particle Physics, University of Geneva, CH-1211, Switzerland

${ }^{6}$ Dipartimento di Matematica e Fisica E. De Giorgi, Universita' del Salento, I-73100, Lecce, Italy

${ }^{7}$ Istituto Nazionale di Fisica Nucleare (INFN) - Sezione di Lecce, I-73100, Lecce, Italy

${ }^{8}$ Institute of High Energy Physics, Chinese Academy of Sciences, Yuquan Road 19B, Beijing 100049, China

${ }^{9}$ University of Chinese Academy of Sciences, Yuquan Road 19A, Beijing 100049, China

${ }^{10}$ Key Laboratory of Dark Matter and Space Astronomy, Purple Mountain Observatory, Chinese Academy of Sciences, Nanjing 210023 , China

${ }^{11}$ School of Astronomy and Space Science, University of Science and Technology of China, Hefei 230026, China

${ }^{12}$ Istituto Nazionale di Fisica Nucleare (INFN) - Sezione di Perugia, I-06123 Perugia, Italy

${ }^{13}$ Institute of Modern Physics, Chinese Academy of Sciences, Nanchang Road 509, Lanzhou 730000, China

${ }^{14}$ National Space Science Center, Chinese Academy of Sciences, Nanertiao 1, Zhongguancun, Haidian district, Beijing 100190, China

${ }^{15}$ Istituto Nazionale di Fisica Nucleare (INFN) - Sezione di Bari, I-70125, Bari, Italy

${ }^{16}$ Dipartimento di Fisica "M. Merlin" dell'Universita' e del Politecnico di Bari, I-70126, Bari, Italy

${ }^{17}$ Department of Physics and Laboratory for Space Research, the University of Hong Kong, Pok Fu Lam, Hong Kong SAR, China

${ }^{1}$ Now at Universita‘ di Sassari, Dipartimento di Chimica e Farmacia, I-07100, Sassari, Italy.

${ }^{2}$ Now at Universita“ degli Studi di Palermo, Dipartimento di Fisica e Chimica "E. Segre"”, via delle Scienze ed. 17, I-90128 Palermo, Italy.

${ }^{3}$ Also at Institute of Physics, Ecole Polytechnique Federale de Lausanne (EPFL), CH-1015 Lausanne, Switzerland.

${ }^{4}$ Also at School of Physics and Electronic Engineering, Linyi University, Linyi 276000, China. 